\title{
Obituary
}

\section{A Tribute to Oleg Zatsarinny (1953-2021): His Life in Science}

\author{
Klaus Bartschat ${ }^{1, *(\mathbb{D}, \text { Charlotte Froese Fischer }}{ }^{2, *(\mathbb{D})}$ and Alexei N. Grum-Grzhimailo ${ }^{3, *(\mathbb{D})}$ \\ 1 Department of Physics and Astronomy, Drake University, Des Moines, IA 50311, USA \\ 2 Department of Computer Science, University of British Columbia, 2366 Main Mall, \\ Vancouver, BC V6T1Z4, Canada \\ 3 Skobeltsyn Institute of Nuclear Physics, Lomonosov Moscow State University, 119991 Moscow, Russia \\ * Correspondence: klaus.bartschat@drake.edu (K.B.); cff@cs.ubc.ca (C.F.F.); grum@sinp.msu.ru (A.N.G.-G.)
}

check for updates

Citation: Bartschat, K.; Fischer, C.F.; Grum-Grzhimailo, A.N. A Tribute to Oleg Zatsarinny (1953-2021): His Life in Science. Atoms 2021, 9, 53. https:// doi.org/10.3390/atoms 9030053

Academic Editor: James F. Babb

Received: 16 July 2021

Accepted: 22 July 2021

Published: 5 August 2021

Publisher's Note: MDPI stays neutral with regard to jurisdictional claims in published maps and institutional affiliations.

Copyright: (c) 2021 by the authors. Licensee MDPI, Basel, Switzerland. This article is an open access article distributed under the terms and conditions of the Creative Commons Attribution (CC BY) license (https:// creativecommons.org/licenses/by/ $4.0 /)$.
Oleg Ivanovich Zatsarinny (Figure 1) was born in the city of Uzhgorod, Ukraine, on 4 November 1953.

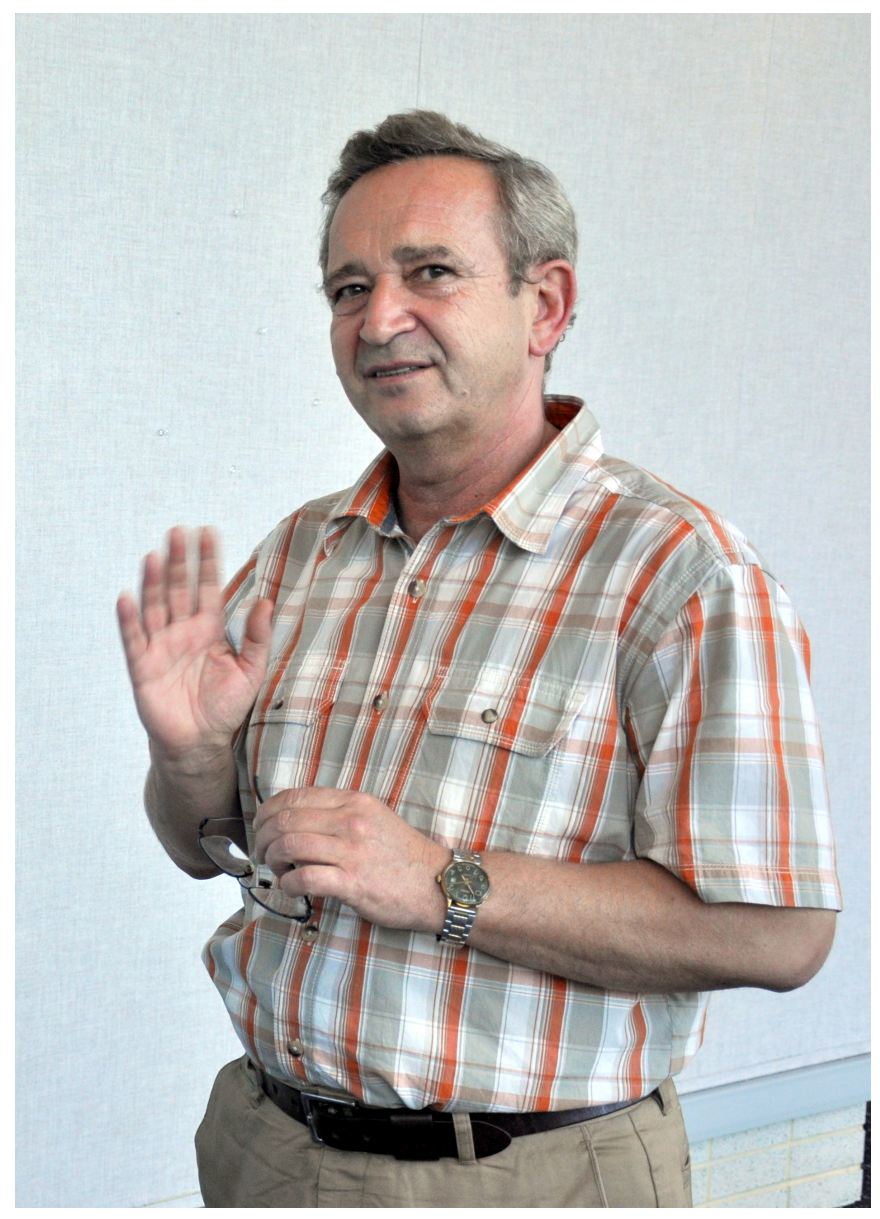

Figure 1. Oleg Zatsarinny (2012). Photograph from private collection of Charlotte Froese Fischer.

As a young boy, Oleg was one of the best students in mathematics and physics in Uzhgorod. He entered the Moscow Institute of Physics and Technology (MIPT) in 1971. Upon graduating with an MSc degree in 1977 under the supervision of E.L. Nagaev and a thesis with the title "Inhomogeneous Ferromagnetism of Conducting Magnets with Ions in a Singlet Ground State", Oleg obtained a position at Uzhgorod University, first in the Department of Physical and Quantum Electronics and then in Theoretical Physics. There, he specialized in calculations for atomic physics. Under the direction of V.I. Lengyel 
(also known as V.I. Lendel), he obtained a PhD degree in 1985 for the thesis "Study of the Autoionization States of Alkaline and Alkaline Metal Atoms and Their Effects on Recombination and Photoionization Processes", which he defended at Vilnius University, and was soon appointed as Head of the Theoretical Physics Division, Laboratory of Electron Collision Physics, Uzhgorod State University. After the organization of a new institute in Uzhgorod, he became a senior research associate at the Institute of Electron Physics of the Ukrainian Academy of Science, a position he would hold from 1993 to 2001. In 1995, he co-authored a book on elementary processes in plasmas [1]. It contained many of his results on electronion collision cross sections. With V.I. Lengyel and E.Y. Remeta as authors, and Oleg as the editor, they published the book, "Electron Scattering on Complex Atoms (Ions)" [2]. With these achievements, Oleg gained international recognition and was able to obtain support for international collaborations and attended international conferences.

When the Soviet Union collapsed in 1991, the financier and philanthropist George Soros played a major role in keeping afloat the scientific communities of Russia and the other former Soviet republics by setting up the International Science Foundation (ISF). At the same time, both the European Union and the Open Society Foundations established foundations to promote cooperation between scientists from western Europe and the independent states of the former Soviet Union. Oleg was successful in getting support from all three sources and soon started a collaboration with Werner Mehlhorn at the University of Freiburg in Germany. Collaborating with the Freiburg group, Oleg explained complex Auger electron spectra produced in pioneering experiments on electron scattering from laser excited atoms.

In the United States, the National Science Foundation established a similar program supporting the collaboration of US scientists with scientists of the former Soviet Union. Due to his research on non-orthogonal orbitals, Charlotte Froese Fischer invited him to Vanderbilt University in 1997, where she introduced him to B-splines and shared her B-spline library of computer programs with him.

In May 2000, Oleg, together with his wife Tatyana, immigrated to the United States. He had accepted a position with Swaraj S. Tayal at Clark Atlanta University who had similar interests in computational physics and laboratory astrophysics. Though the appointment was only for 15 months, Oleg continued as a research consultant for 15 years. He then spent 2001-2003 at Western Michigan University at the Department of Physics with Thomas W. Gorczyca, working on electron collisions mostly of interest to plasma physics and astrophysics.

In October 2003, Oleg moved to Des Moines, Iowa, as a Senior Researcher at Drake University, where he stayed until the end of his life on 2 March 2021 and collaborated with the group of Klaus Bartschat. They co-authored 126 papers together, including a 2013 Topical Review on the B-spline R-matrix method [3].

In 2006, Oleg published "BSR: B-spline atomic R-matrix codes" in Computer Physics Communications [4]. This code used the R-matrix method to calculate electron-atom and electron-ion collision processes, with options to calculate radiative data, photoionization, etc., and to perform these calculations either in an LS-coupling or intermediate-coupling scheme that could include Breit-Pauli terms in the Hamiltonian. This is his most-cited paper among those where Oleg was first author. In the relativistic version, however, a problem arose, because spurious solutions affected the R-matrix that joins the outer and inner regions. This problem was solved by expressing large and small components by splines of different orders [5]. The resulting Dirac R-matrix version of BSR (unpublished) and the DBSR_HF program [6] are among the most computationally accurate programs dealing with complex atoms and ions available at this time.

In 2008, the Division of Atomic, Molecular and Optical Physics of the American Physical Society awarded Oleg the status of "Fellow" for "the development of the B-Spline R-matrix method with non-orthogonal orbital sets for atomic structure calculations of exceptional accuracy and benchmark calculations for excitation and ionization of complex atoms and ions by photon and electron impact". 
Oleg was the principal or co-principal investigator for a number of National Science Foundation grants. In later years, he also became an educator. In addition to teaching existing courses, Oleg introduced the Computational Physics course into the curriculum at Drake University. He published over 200 papers in peer-reviewed journals, presented 20 invited talks at international meetings, and contributed several hundred papers. His work has already been cited over 5000 times and will continue to influence atomic physics for decades. The latest version of his BSR and DBSR codes are freely available at Oleg's GitHub repository [7]. The BSR version is also being preserved through the Atomic and Molecular Physics Gateway [8], where the source code as well as many sample input files and run scripts for test cases are made available to all interested users.

Conflicts of Interest: The authors declare no conflict of interest.

\section{References}

1. Borovsky, A.V.; Zapryagaev, S.A.; Zatsarinny, O.I.; Manakov, N.L. Plasma of Multicharged Ions. Elementary Processes, Kinetics and $X$-Ray Lasers; Khimiya: Saint-Petersburg, Russia, 1995. (In Russian)

2. Lengyel, V.; Lendel, V.I.; Remeta, E.Y. Electron Scattering on Complex Atoms (Ions); Zatsarinny, O., Ed.; Horizons in World Physics; Nova Science Publishers, Inc.: London, UK, 2000; Volume 234, p. 474.

3. Zatsarinny, O.; Bartschat, K. The B-spline R-matrix method for atomic processes: Application to atomic structure, electron collisions and photoionization. J. Phys. B At. Mol. Opt. Phys. 2013, 46, 112001. [CrossRef]

4. Zatsarinny, O. BSR: B-spline atomic R-matrix codes. Comp. Phys. Commun. 2006, 174, 273. [CrossRef]

5. Froese Fischer, C.; Zatsarinny, O. A B-spline Galerkin method for the Dirac equation. Comp. Phys. Commun. $2009,180,879$. [CrossRef]

6. Zatsarinny, O.; Froese Fischer, C. A B-spline Dirac-Hartree-Fock program. Comp. Phys. Commun. 2016, 202, 287. [CrossRef]

7. Oleg Zatsarinny's GitHub Repository. Available online: https:/ / github.com/zatsaroi/ (accessed on 16 July 2021).

8. Atomic and Molecular Physics Gateway. Available online: https://ampgateway.org/ (accessed on 16 July 2021). 